\title{
Hans-Christoph Askani, Carlos Mendoza, Denis Müller, Dimitri Andronicos (éd.), Où est la vérité ? La théologie aux défis de la Radical Orthodoxy et de la déconstruction
}

Genève, Labor et Fides, coll. « Lieux théologiques », 2012,368 p.

\section{Daniel Vidal}

\section{(2) OpenEdition} Journals

\section{Édition électronique}

URL : http://journals.openedition.org/assr/26280

DOI : $10.4000 /$ assr.26280

ISSN : 1777-5825

Éditeur

Éditions de l'EHESS

\section{Édition imprimée}

Date de publication : 31 décembre 2014

Pagination : 114

ISBN : 978-2-7132-2467-6

ISSN : 0335-5985

\section{Référence électronique}

Daniel Vidal et Daniel Vidal, « Hans-Christoph Askani, Carlos Mendoza, Denis Müller, Dimitri Andronicos (éd.), Où est la vérité ? La théologie aux défis de la Radical Orthodoxy et de la déconstruction », Archives de sciences sociales des religions [En ligne], 168 | 2014, mis en ligne le 31 mars 2015, consulté le 22 septembre 2020. URL : http://journals.openedition.org/assr/26280 ; DOI : https://doi.org/10.4000/assr.26280

Ce document a été généré automatiquement le 22 septembre 2020.

(ㄷ) Archives de sciences sociales des religions 
Hans-Christoph Askani, Carlos Mendoza, Denis Müller, Dimitri Andronicos (éd.), Où est la vérité ? La théologie aux défis de la Radical Orthodoxy et de la déconstruction

Genève, Labor et Fides, coll. « Lieux théologiques », 2012,368 p.

Daniel Vidal

\section{RÉFÉRENCE}

Hans-Christoph Askani, Carlos Mendoza, Denis Müller, Dimitri Andronicos (éd.), Où est la vérité ? La théologie aux défis de la Radical Orthodoxy et de la déconstruction, Genève, Labor et Fides, coll. « Lieux théologiques », 2012,368 p. 
La vérité, cet impensable anthropologique par son singulier même, s'entend ici sous condition d'un contexte théologique soumis depuis plusieurs décennies à une double contestation. La première, venue du plus profond de son héritage, se définit comme Radical Orthodoxy, dont les traités multipliés de John Milbank exposent les thèses centrales avec rigueur et pugnacité, notamment dans ce qui apparaît comme son ouvrage majeur, Théologie et théorie sociale (1990, trad. fr. 2006). Contre l'éclatement relativiste de la modernité, aggravé par le saut en postmodernité, cette nouvelle théologie se livre à une critique radicale de la raison séculière, et réclame une restauration massive des fondamentaux de la pensée chrétienne. La seconde contestation advient dans le contexte de la post-

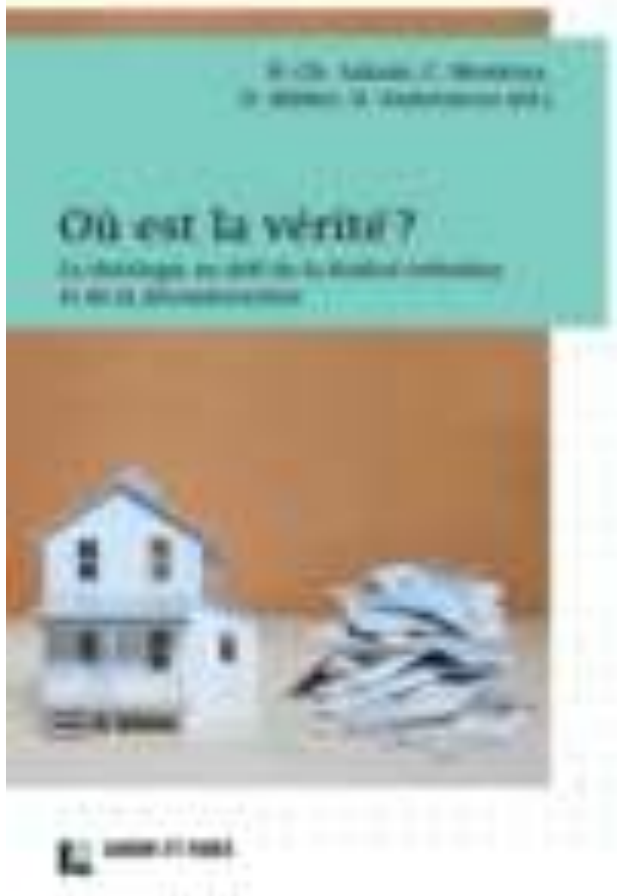
modernité, et comme sa conceptualisation privilégiée. La philosophie de la déconstruction remet en cause le caractère absolu de la vérité, insistant sur sa dissémination infinie, et propose une ontologie "prise dans le jeu infini de la différance, de la trace, d'une absence qui habite toujours la présence ». C'est du moins en ces termes que Milbank définit la pensée de Derrida, pour s'y opposer frontalement. Les enjeux de cette dispute théologique sont considérables. La théologie va-t-elle re-devenir une nouvelle orthodoxie incapable de prendre en compte ce qui relève de l'historicité du sujet humain? Qu'en est-il de la « raison »? Qu'en est-il de l'autonomie du sujet? Qu'en est-il de l'altérité et de la foi comme expérience existentielle ? Etc.

Milbank se situe au centre d'une polémique qui peut décider, pour la théologie, d'un déplacement en effet radical de ses assises. Pour le théologien anglais, dont on peine à identifier ce qui relève d'un anglicanisme décalé, d'un protestantisme sublimé, ou d'un catholicisme refoulé, il n'est de théologie que fondée sur «l'infinité originelle de la raison ", au-delà du pensable et du connaissable, " raison inconnue ", " mystère ». Une « raison" qui est à vrai dire "l'Autre de la raison ", qui implique aussitôt "l'idée de transcendance». C'est par cette "raison originelle» que notre propre pouvoir de raisonner "s'enracine dans la réalité ». Une fois "donnée la transcendance ", écrit Milbank, « on n'a pas à choisir entre la priorité de la raison et la priorité de l'être », c'est-à-dire entre ce qui relève du connu et ce qui ressortit de l'inconnaissable. D'autant moins que «la raison elle-même est un don dérivant de l'infinité de la raison inconnue ». Retour donc au "mystère ». Là se formule sans doute la critique la plus radicale des accomplissements de la raison séculière, qui, pour Milbank, ne se conçoit que sous condition de transcendance, mais dans une séparation radicale avec cet absolu. Pierre Gisel note très justement que le protestantisme pense en même temps l'hétérogénéité absolue de Dieu et la radicalité existentielle, de même que, pour Milbank, le sujet humain est vu « comme se tenant en vis-à-vis de Dieu, et séparé ». 
Mais le théologien anglais rate ce que P. Gisel appelle "la dramatique de la liberté inscrite au cœur de la modernité », qui suppose qu'une médiation s'établisse entre "l'humain et l'altérité », sous peine de vivre cette «dramaturgie » comme scénario de la désespérance. Calvin "instituait» le Christ comme figure "archétypale de la médiation » au centre de la création.

C'est au nom de ce rappel constant à la thématique du sujet engagé en historicité, « aux prises avec le réel ", que la «Radical Orthodoxy» est questionnée par l'ensemble des contributeurs de l'ouvrage, redoutant pour certains un "retour à la chrétienté " comme base d'organisation sociale (Xavier Gravend-Tirole), ou la formulation d'une théologie politique "résolument évangélique " (Shafique Keshavjee), qui ignorerait la différenciation du politique et du religieux. L'absolutisation de la vérité, on le sait, fonde un régime de violence intellectuelle et sociale. Parce qu'elle réduit la modernité à des séries de distinctions bipolaires insoutenables (foi/raison, grâce/nature, etc.), la théologie radicale de Milbank et MacIntyre se présente comme seule "science" capable, si l'on peut dire, de transcender ces « apories de la raison moderne fondée sur ces dualismes" (Dimitri Andronicos). Quand il convient au contraire de libérer continûment l'espace d'une éthique de la responsabilité dans le régime d'une ontologie où «le dépouillement et l'absence sont originaires et indépassables». Contestation décisive de la théologie milbankienne : on n'est plus dans le registre de la vérité absolue surplombant un monde qu'elle enchanterait, mais dans le vif d'une existence humaine où toute altérité est au fondement de soi, et où le don et le pardon, pour reprendre l'analyse de Derrida, ne sont possibles que détachés de toute raison «économique ». La gratuité sera cette grâce. Qui, loin de dispenser de tout engagement, dispose au contraire le sujet sous l'impératif éthique d'une responsabilité plénière - pourrait-on dire originelle? Résumant la thèse derridienne, Andronicos écrit : « Si nous ne sommes pas responsables de tout, si nous devons répondre uniquement de ce que nous connaissons, de ce que nous pouvons ou avons fait, alors nous ne sommes pas responsables. Il faut répondre de tout devant l'absolu pour devenir pleinement sujet de responsabilité ». C'est à l'évidence cette réappropriation, par le sujet humain, de la responsabilité et de la liberté qui l'autorise, qui marque la frontière infranchissable entre la conception « moderne » de l'homme en son histoire et son temps propres - et la théologie systématique et conservatrice de Milbank. Conception "moderne " qui peut en effet s'articuler à une théologie fondée non plus sur un corps de doctrine, mais, selon la proposition de François Dermange, «sur la Bible [...] comme expérience existentielle ». Une théologie de la libération, ainsi qu'une théologie politique, peuvent alors se proposer, qui s'enracinent l'une et l'autre dans une historicité singulière.

4 Voulant restaurer un discours théologique "comme matrice des autres discours ", la Radical orthodoxy considère l'autonomie moderne comme « une apostasie par rapport à la théonomie chrétienne ». Et sans doute l'élément mystique est-il l'événement le plus décisif dans la formulation moderne du sujet en religion. Par là, en effet, en suivant l'argumentaire de Bergson, s'opère ce que Anthony Feneuil appelle «l'identification de l'absolu à une expérience ». Que ce nouveau « sujet » soit l'un quelconque des humains, ou l'humanité en son universalité, du moins est-il assuré de faire «l'expérience du réel en son fond». Alors ce "fond du monde » réel parle de cet «absolu» comme de son langage originel. Pour le philosophe bergsonien, le christianisme est peut-être «la religion des mystiques complets ", et «sujet de l'expérience mystique complète ». À coup sûr la mystique est-elle cette insurrection du sujet sur les décombres de l'Unité perdue, ainsi qu'elle se présente dans le long temps qu'analyse Michel de Certeau. Est- 
ce un hasard si l'ouvrage convoque ici la mystique ? Si la « liberté » du sujet est requise contre l'imposition de la théologie "radicale " ? Les auteurs maintiennent le temps de Dieu et le temps des hommes: «Deux temps se touchent, se heurtent et se transforment - le temps de notre humanité et le temps de Dieu pour l'homme ", écrit Hans-Christoph Askani. C'est dans ce «heurt » et cette "touche » que la mystique et son sujet s'accomplissent, dans ce "jeu comme liberté et comme temps ». Aussi bien, après avoir traversé l'épreuve d'une orthodoxie systémique, faut-il en venir à ce qui s'oppose à la radicalité antimoderne, soit à cette « chose » de stricte subjectivité, mais aussitôt de partage global, "la chose même de la foi » - cette expérience extrême autour de laquelle s'organise la réflexion de Carlos Mendoza. Telle foi s'instaure dans une figure du christianisme que Jean-Luc Nancy appelle la "déclosion», quand c'est l'absence de Dieu «qui fait proprement divinité, dont le vide-de-divinité est proprement la vérité ». Foi qui se dit "événement de gratuité et donation», seul capable, dans le moment religieux d'une postmodernité délivrée de ses caricatures et de ses censeurs, d'évoquer « la consistance non intentionnelle du réel » et de lui donner sens. Au côté d'autres voix venues d'autres raisons et d'autres sources. 